\title{
Migraine in midlife could be connected to increased risk of late-life parkinsonism
}

People who have migraine in middle age could be at increased risk of developing Parkinson disease (PD) or parkinsonian symptoms later in life, according to a longitudinal study comprising 5,620 adults from Reykjavik, Iceland.

\section{People who were} experiencing migraine attacks ... were more likely to be diagnosed with PD ... in later life... 77

"Because previous studies have linked migraine with aura to increased risk of stroke, there has been growing interest in whether migraine might be linked with other neurological symptoms," explains Ann Scher, the lead author of the study. "To our knowledge, this is the first study to link migraine with parkinsonism or PD in the general population."

Study participants were interviewed about migraine symptoms in middle age (mean 51 years). About 25 years later, the same participants were interviewed about whether they had been diagnosed with PD, experienced symptoms of parkinsonism or restless leg syndrome, or had a family history of PD. The investigators also examined inpatient medical records and administrative records of medication use. "People who were experiencing migraine attacksparticularly migraine with aura-at the time of the first interview were more likely to be diagnosed with PD or to have parkinsonian symptoms in later life than people who did not have headache in midlife," summarizes Scher.

Migraine with aura in middle age was associated with a two-fold increase in risk of $\mathrm{PD}$, but Scher points out that the risk of $\mathrm{PD}$ in people with migraine is still very low $(<2.5 \%)$. About $20 \%$ of the people who had migraine with aura-but only $8 \%$ of those without migraine-reported having four or more parkinsonian symptoms.
Scher emphasizes that the researchers controlled for use of migraine medications, some of which are dopamine antagonists. Therefore, a causal link between migraine pharmacotherapy and development of PD seems unlikely.

A mechanism that increases vulnerability to both migraine and parkinsonism, such as a shared genetic risk factor or dysfunction of the dopaminergic system, might explain the observed association. Another potential predisposing factor could be a history of head injury, which was reported more often in people with migraine with aura than in those without. In the future, understanding the shared risk factors might aid the identification of individuals at risk of PD.

\section{Hemi Malkki}

Original article Scher, A. I. et al. Midlife migraine and late-life parkinsonism: AGES-Reykjavik Study. Neurology doi:10.1212/WNL.000000000000084 